\title{
Elastic Modulus Imaging: Some exact solutions of the compressible elastography inverse problem
}

\author{
Paul E. Barbone and Assad A. Oberai $\ddagger$ \\ Aerospace \& Mechanical Engineering, Boston University, 110 Cummington St., \\ Boston, MA 02215, US \\ E-mail: barbone@bu.edu, oberaa@rpi.edu
}

\begin{abstract}
We consider several inverse problems motivated by elastography. Given the (possibly transient) displacement field measured everywhere in an isotropic, compressible, linear elastic solid, and given density $\rho$, determine the Lamé parameters $\lambda$ and $\mu$. We consider several special cases of this problem: (a) For $\mu$ known apriori, $\lambda$ is determined by a single deformation field up to a constant. (b) Conversely, for $\lambda$ known apriori, $\mu$ is determined by a single deformation field up to a constant. This includes as a special case that for which the term $\lambda \nabla \cdot \boldsymbol{u} \equiv 0$. (c) Finally, if neither $\lambda$ nor $\mu$ is known apriori, but Poisson's ratio $\nu$ is known, then $\mu$ and $\lambda$ are determined by a single deformation field up to a constant. This includes as a special case plane stress deformations of an incompressible material. Exact analytical solutions valid for $2 D$, $3 D$, and transient deformations are given for all cases in terms of quadratures. These are used to show that the inverse problem for $\mu$ based on the compressible elasticity equations is unstable in the limit $\lambda \rightarrow \infty$. Finally, we use the exact solutions as a basis to compute nontrivial modulus distributions in a simulated example.
\end{abstract}

$\ddagger$ Department of Mechanical, Aerospace and Nuclear Engineering, Rensselaer Politechnic Institute, Troy, NY 12180 


\section{Introduction}

Physicians often detect and diagnose tissue abnormalities by manual palpation, i.e. by touch. Palpation is an effective diagnostic tool because tissue mechanical properties, including stiffness, are affected by pathology. Elastography refers to the imaging of soft tissue on the basis of (shear) elastic modulus. As such, elastography aims to construct visual images showing the spacial distribution of the physical properties that might otherwise be sensed by feel by the physician. Furthermore, the potential exists within elastography to quantify those properties. In this work we are concerned with quantifying the (linear) elastic shear modulus of soft tissue via elastography.

The shear elastic modulus of different tissues can differ by orders of magnitude [1]. Therefore, we expect that different tissues will be clearly distinguished with high contrast in a shear modulus image. The inherent contrast in material properties that underlies mammography or sonography, on the other hand, is only a few percent. That is, the speed of sound in soft tissues is roughly $c \approx 1540 \mathrm{~m} / \mathrm{s}$, with typically only $2-3 \%$ and at most about $10 \%$ deviation from this value [2]. Similarly, the mass density of soft tissues is very close to that of water [2].

All elastography techniques rely on being able to image soft tissue while it is being deformed by a set of applied forces. Through image processing, the displacement (or sometimes velocity) field everywhere in the region of interest is inferred. An inverse problem for the elastic modulus is formulated, given the measured displacement fields, an assumed form of the tissue's constitutive equation (e.g. linear elastic), and the law of conservation of momentum. In many cases, further assumptions are made in order to simplify the problem. We point out that the availability of interior data leads to an inverse problem with considerably different character than one in which only boundary data are given.

The purpose of this paper is to document the existence of several exact analytical solutions of the inverse elasticity problem. The mathematics that leads to these solutions is elementary, and the evaluation of the solutions themselves is straightforward. That the existence of the exact analytical solutions presented here may not be well known is evident in the recent explosion of comparatively cumbersome and complicated algorithms published to solve the same inverse problem.

In this paper we study tissue models based on isotropic, compressible, linear elasticity. We present the exact solution of the inverse elasticity problem under various assumptions that have been made by other researchers. Our solutions are valid for $2 D$ and $3 D$ deformations, for both quasi-static and transient deformations, and treats the entire vector elasticity equation. The special cases we consider are: (a) For $\mu$ known apriori, $\lambda$ is determined by a single deformation field up to a constant. (b) Conversely, for $\lambda$ known apriori, $\mu$ is determined by a single deformation field up to a constant. A special case of (b) is that for which $\lambda \nabla \cdot \boldsymbol{u} \equiv 0$. (c) Also, if neither $\lambda$ nor $\mu$ is known apriori, but Poisson's ratio $\nu$ is known, then $\mu$ is determined by a single deformation field up to a constant. This includes as a special case plane stress deformations of an 
incompressible material.

The availability of the exact solutions presented here are expected to be useful for several purposes. First, the exact solutions facilitate the mathematical analysis of the inverse problem. For example, a result related to the stability of the compressible inverse problem in the limit of large $\lambda$ (the second Lamé parameter) is obtained in section 8 , below. As another example that is not pursued below, having the exact solution allows predictions of the noise statistics of reconstructions from noise statistics of the data. Secondly, the exact solution may be directly useful for inversion in applications where the entire displacement vector can be measured in a compressible material. Third, the exact solution applies to the situation of plane stress loading of an incompressible material. In that case, only two components of displacement are needed in the plane; see section 6 below. Finally, the exact solution may be useful in benchmarking other computational approaches based, for example, on iterative optimization $[3,4,5,6,7]$.

On the other hand, the exact solutions presented below have several shortcomings that may limit their practical use. First, they are based on compressible elasticity, and therefore lead to unstable reconstructions when used near the incompressible limit. Second, they require the full $3 D$ displacement vector (or $2 D$ displacement vector in $2 D$ problems) to be measured everywhere. Third, they require derivatives of strain, which may lead to impractically high noise amplification in practice. Nevertheless, a solvability condition presented below may provide a practical means to evaluate whether a given measured strain field is "too noisy" to give a reliable modulus estimate in practice.

While MR can provide all three displacement components in $3 D$, ultrasound is currently challenged to provide precise displacement measurements in directions orthogonal to the direction of sound propagation. The availability of simple, exact, modulus reconstruction formulas presented here may provide impetus for the development of novel ultrasound acquisition techniques to measure the full displacement vector.

In the next section, we set up the forward compressible and incompressible elasticity equations. Following that, we solve these for the special cases described above. For each case we present a solvability condition that must be satisfied by the given data. When this solvability condition is violated, no single-valued solution exists. The special case of

plane stress deformations of an incompressible material warrants its own section, and is treated next. Following that, we use the exact solutions to demonstrate the instability of the shear modulus inversion when $\lambda \rightarrow \infty$. Finally, we use the exact solutions as a basis to compute nontrivial modulus distributions in simulated examples.

\section{Formulation: Forward compressible and incompressible elasticity problems}

We consider an elastic solid occupying the region $\Omega$, with boundary $\Gamma$. We let $\boldsymbol{u}(x, y, z, t) \equiv \boldsymbol{u}\left(x_{1}, x_{2}, x_{3}, t\right) \equiv \boldsymbol{u}(\boldsymbol{x}, t)$ denote the displacement field as a function of the spatial coordinate $\boldsymbol{x}$ and time $t$. The linearized strain components, measured with 
respect to Cartesian axes, are given by

$$
\epsilon_{i j}=\frac{1}{2}\left[\partial_{i} u_{j}+\partial_{j} u_{i}\right]
$$

In (1) we have introduced the shorthand notation $\partial_{j}=\frac{\partial}{\partial x_{j}}$.

The balance of linear momentum over each part of the material is given by

$$
\sum_{j=1}^{3} \partial_{j} \sigma_{i j} \equiv \partial_{j} \sigma_{i j}=\rho \partial_{t t} u_{i}
$$

In writing (2) we have neglected all body forces and adopted the summation convention over repeated indices, which we will continue to use throughout the paper.

Equations (1) and (2) apply to both compressible and incompressible materials. The stress-strain relation, or constitutive equation can be written as:

$$
\sigma_{i j}=-p \delta_{i j}+2 \mu \epsilon_{i j}
$$

In (3), $\sigma_{i j}$ is a component of the stress tensor, $\boldsymbol{\sigma} ; \mu$ is the material shear modulus, which in some texts is denoted by $G$. It is related to Young's modulus $E$ and Poisson's ratio $\nu$ through $\mu=G=E / 2(1+\nu)$ [8]. For an incompressible material, $\nu=1 / 2$. In addition to equation (3), we have:

$$
\sum_{k=1}^{3} \epsilon_{k k} \equiv \epsilon_{k k} \equiv \partial_{k} u_{k}=-p / \lambda
$$

In equation (4), $\lambda$ is the second Lamé constant. It is related to $\mu$ and $\nu$ by $\lambda=$ $2 \mu \nu /(1-2 \nu)[8]$. We note that the average normal stress is $\sigma_{k k} / 3=-p+2 \mu \epsilon_{k k}$.

For incompressible materials, we take $\lambda \rightarrow \infty$ in (4) with $p$ finite to get the incompressibility constraint, expressed as

$$
\epsilon_{k k}=\partial_{k} u_{k}=0 \text {. }
$$

In this case, $p$ in (3) can be interpreted as a pressure field in the solid.

Equation (3) can be substituted into equation (2) to write the momentum equation directly in terms of $p$ and the displacement $\boldsymbol{u}$ :

$$
-\partial_{i} p+\partial_{j}\left(\mu \partial_{i} u_{j}\right)+\partial_{j}\left(\mu \partial_{j} u_{i}\right)=\rho \partial_{t t} u_{i}
$$

Equations (6) and (4) (or its limiting form (5)) are sufficient to define the pressure and

displacement fields everywhere in the solid, when $\lambda, \mu$, and $\rho$ are known and appropriate boundary conditions are specified.

\section{Case (a): Exact Solution for $\lambda(\boldsymbol{x})$ Given $\mu(\boldsymbol{x})$}

\subsection{Inverse problem statement}

This is the simplest of all cases we consider, though it hasn't been specifically treated before in the literature. We note its solution here for completeness. The static (resp. dynamic) inverse problem with which we are concerned is the following: Given $\boldsymbol{u}(\boldsymbol{x})$ (or 
$\boldsymbol{u}(\boldsymbol{x}, t)), \mu(\boldsymbol{x})$, and $\rho(\boldsymbol{x})$ defined on $\Omega(\Omega \times(0, T>0))$, find $\lambda(\boldsymbol{x})$ such that equations (6) and (4) are satisfied.

We note that (6) can be rewritten in terms of strain $\boldsymbol{\epsilon}$ using (1) to get

$$
-\nabla(\lambda \nabla \cdot \boldsymbol{u})+2 \nabla \cdot(\mu \boldsymbol{\epsilon})=\rho \partial_{t t} \boldsymbol{u} .
$$

Further, given $\boldsymbol{u}$, we may use (1) to determine $\boldsymbol{\epsilon}$. Thus we may consider $\boldsymbol{\epsilon}$ known.

\subsection{Solution}

In the case $\nabla \cdot \boldsymbol{u} \neq 0$, equation (7) may be integrated directly to yield:

$$
\lambda(\boldsymbol{x})=\frac{1}{\nabla \cdot \boldsymbol{u}} \int_{\boldsymbol{x}_{o}}^{\boldsymbol{x}}\left[2 \nabla \cdot\left(\mu\left(\boldsymbol{x}^{\prime}\right) \boldsymbol{\epsilon}\left(\boldsymbol{x}^{\prime}, t\right)\right)-\rho \partial_{t t} \boldsymbol{u}\left(\boldsymbol{x}^{\prime}, t\right)\right] \cdot d \boldsymbol{x}^{\prime}+\lambda\left(\boldsymbol{x}_{o}\right) .
$$

The integral in (8) is independent of the path taken from $\boldsymbol{x}_{o}$ to $\boldsymbol{x}$ provided the integrand (i.e. the given data) satisfies an integrability condition. For a simply connected domain, this condition is:

$$
\nabla \times\left[2 \nabla \cdot\left(\mu\left(\boldsymbol{x}^{\prime}\right) \boldsymbol{\epsilon}\left(\boldsymbol{x}^{\prime}\right)\right)-\rho \partial_{t t} \boldsymbol{u}\right]=0 .
$$

Thus if (9) is not satisfied by the data, a single valued solution of (7) for $\lambda(\boldsymbol{x})$ does not exist for that data.

\section{Case (b): Exact Solution for $\mu(\boldsymbol{x})$ Given $\lambda(\boldsymbol{x})$.}

Based on the emperical evidence [2] that density and sound speed are roughly constant across soft tissues, one might reasonably assume that $\rho$ and $\lambda$ are both known and constant. Here we assume that $\rho$ and $\lambda$ are known, but not necessarily constant. Note that the special case $\lambda \nabla \cdot \boldsymbol{u}=0$ considered in follows from our solution by setting $\lambda=0$. That special case is considered in $[9,10,11,12]$, but our solution differs from the others in two respects: we keep the term $\nabla \boldsymbol{u} \cdot \nabla \mu$, neglected in $[9,10,11,12]$, and we keep the term $\nabla \mu \cdot \nabla \boldsymbol{u}$, further neglected in $[9,10,11]$. Nevertheless, the exact solution is

available without these simplifications, but in the form given here requires the entire displacement vector to be known.

\subsection{Inverse problem statement}

Given $\boldsymbol{u}(\boldsymbol{x})$ (or $\boldsymbol{u}(\boldsymbol{x}, t)), \lambda(\boldsymbol{x})$, and $\rho(\boldsymbol{x})$ defined on $\Omega(\Omega \times(0, T>0))$, find $\mu(\boldsymbol{x})$ such that equations (6) and (4) are satisfied.

\subsection{Solution}

We use (4) in (7) and move known terms to the right hand side to obtain:

$$
\begin{aligned}
2 \mu \nabla \cdot \boldsymbol{\epsilon}+2 \boldsymbol{\epsilon} \nabla \mu & =F_{b}(\boldsymbol{x}, t), \\
F_{b}(\boldsymbol{x}, t) & =\rho \partial_{t t} \boldsymbol{u}-\nabla(\lambda \nabla \cdot \boldsymbol{u})
\end{aligned}
$$


4.2.1. Particular solution We let $\mu_{h}(\boldsymbol{x})$ be a solution of equation (10) with $F_{b}$ set equal to zero. Then setting $\mu=\mu_{p}=f(\boldsymbol{x}) \mu_{h}(\boldsymbol{x})$ in (10) gives

$$
\left[2 f \mu_{h} \nabla \cdot \boldsymbol{\epsilon}+2 f \boldsymbol{\epsilon} \nabla \mu_{h}\right]+2 \mu_{h} \boldsymbol{\epsilon} \nabla f=F_{b}(\boldsymbol{x}, t)
$$

The term in brackets in (12) is zero since $\mu_{h}$ is a homogeneous solution of (10), yielding

$$
2 \mu_{h} \epsilon \nabla f=F_{b}(\boldsymbol{x}, t) \text {. }
$$

We may now solve (13) for $\nabla f$ and integrate to find

$$
f(\boldsymbol{x})=\int_{\boldsymbol{x}_{o}}^{\boldsymbol{x}} \frac{1}{2 \mu_{h}\left(\boldsymbol{x}^{\prime}\right)} \boldsymbol{\epsilon}^{-1}\left(\boldsymbol{x}^{\prime}, t\right) F_{b}\left(\boldsymbol{x}^{\prime}, t\right) \cdot d \boldsymbol{x}^{\prime} .
$$

In (14) we assumed (without loss of generality) $f\left(\boldsymbol{x}_{o}\right)=0$. The integration in (14) may be taken along any path from a fixed point $\boldsymbol{x}_{o}$ to the point $\boldsymbol{x}$, where $f(\boldsymbol{x})$ is being evaluated. A particular solution of (10) thus follows:

$$
\mu_{p}(\boldsymbol{x})=\mu_{h}(\boldsymbol{x}) \int_{\boldsymbol{x}_{o}}^{\boldsymbol{x}} \frac{1}{2 \mu_{h}\left(\boldsymbol{x}^{\prime}\right)} \boldsymbol{\epsilon}^{-1}\left(\boldsymbol{x}^{\prime}, t\right)\left[\rho \partial_{t t} \boldsymbol{u}\left(\boldsymbol{x}^{\prime}, t\right)-\nabla\left(\lambda \nabla \cdot \boldsymbol{u}\left(\boldsymbol{x}^{\prime}, t\right)\right)\right] \cdot d \boldsymbol{x}^{\prime} .(15)
$$

We note that (15) remains valid if $\lambda=0$.

4.2.2. Homogeneous solution To find the homogeneous solution of (10) we recall that $\mu>0$, and let $q(\boldsymbol{x})=\log \left(\mu_{h}(\boldsymbol{x})\right)$. The chain rule then gives us $\nabla q=\mu_{h}{ }^{-1} \nabla \mu_{h}$. Substituting into (10) with the right side set equal to zero gives

$$
2 \nabla \cdot \boldsymbol{\epsilon}+2 \boldsymbol{\epsilon} \nabla q=0 .
$$

Solving for $\nabla q$ and integrating yields:

$$
q(\boldsymbol{x})=q\left(x_{o}\right)-\int_{\boldsymbol{x}_{o}}^{\boldsymbol{x}} \boldsymbol{\epsilon}^{-1}\left(\boldsymbol{x}^{\prime}, t\right) \nabla \cdot \boldsymbol{\epsilon}\left(\boldsymbol{x}^{\prime}, t\right) \cdot d \boldsymbol{x}^{\prime} .
$$

Thus we find the homogeneous solution

$$
\mu_{h}(\boldsymbol{x})=\mu\left(\boldsymbol{x}_{o}\right) \exp \left\{-\int_{\boldsymbol{x}_{o}}^{\boldsymbol{x}} \boldsymbol{\epsilon}^{-1}\left(\boldsymbol{x}^{\prime}, t\right) \nabla \cdot \boldsymbol{\epsilon}\left(\boldsymbol{x}^{\prime}, t\right) \cdot d \boldsymbol{x}^{\prime}\right\} .
$$

The total solution is then obtained by summing the homogeneous and particular solutions (15) and (18)

$$
\mu(\boldsymbol{x})=\mu_{h}(\boldsymbol{x})+\mu_{p}(\boldsymbol{x}) .
$$

The total solution requires the specification of $\mu\left(\boldsymbol{x}_{o}\right)$.

The time $t$ enters (19) as a parameter through the dependence of $\mu_{h}(\boldsymbol{x})$ and $\mu_{p}(\boldsymbol{x})$ on $\boldsymbol{\epsilon}(\boldsymbol{x}, t)$ and $\boldsymbol{u}(\boldsymbol{x}, t)$. Of course, any physically meaningful solution for the shear modulus $\mu(\boldsymbol{x})$ must be independent of $t$, and so all time dependence must necessarily drop out of (19) identically.

4.2.3. Solvability Conditions Carrying out the operations above requires two important assumptions that may not be satisfied in practice. First, for $\epsilon^{-1}$ to exist, we need

$$
\operatorname{det} \epsilon \neq 0 \text {. }
$$

Second, as above, for the integral appearing in (17) to be path independent requires the integrand to satisfy:

$$
\nabla \times\left[\boldsymbol{\epsilon}^{-1} \nabla \cdot \boldsymbol{\epsilon}\right]=0 .
$$


5. Case (c): Exact Solution for $\mu(x)$ and $\lambda(x)$ given $\nu$.

Several researchers have modeled the inverse elasticity problem for soft tissues by choosing a constant Poisson's ratio, $\nu$, close to $1 / 2[5,6,7]$. A value of $\nu=1 / 2$ corresponds to perfect incompressibility. A value of $\nu=0.495$ [1] is often cited for soft tissues. Here we present the exact solution for $\nu$ known and constant.

\subsection{Inverse problem statement}

Given $\boldsymbol{u}(\boldsymbol{x})$ (or $\boldsymbol{u}(\boldsymbol{x}, t)$ ), $\nu=$ constant, and $\rho(\boldsymbol{x})$ defined on $\Omega(\Omega \times(0, T>0)$ ), find $\mu(\boldsymbol{x})$ and $\lambda(\boldsymbol{x})$ such that equations (6) and (4) are satisfied, and:

$$
\lambda=\frac{2 \nu}{1-2 \nu} \mu=B \mu .
$$

We note that $\lambda$ becomes infinite when $\nu=1 / 2$.

\subsection{Solution}

By using (22) in (4) and (7) we find

$$
\boldsymbol{A} \mu+\nabla \boldsymbol{A} \nabla \mu=\rho \partial_{t t} \boldsymbol{u}\left(\boldsymbol{x}^{\prime}, t\right) \equiv F_{c}(\boldsymbol{x}, t) .
$$

The matrix $\boldsymbol{A}$ is defined to be:

$$
\boldsymbol{A}=B(\nabla \cdot \boldsymbol{u}) \mathbf{1}+2 \boldsymbol{\epsilon}
$$

Equation (23) may be solved by the same methods as used to solve (10). Repeating those steps thus gives the following particular solution:

$$
\mu_{p}(\boldsymbol{x})=\mu_{h}(\boldsymbol{x}) \int_{\boldsymbol{x}_{o}}^{\boldsymbol{x}} \frac{\rho}{\mu_{h}\left(\boldsymbol{x}^{\prime}\right)}[B(\nabla \cdot \boldsymbol{u}) \mathbf{1}+2 \boldsymbol{\epsilon}]^{-1} \partial_{t t} \boldsymbol{u}\left(\boldsymbol{x}^{\prime}, t\right) \cdot d \boldsymbol{x}^{\prime} .
$$

In (25), $\mu_{h}$ is a solution of (23) with $F_{c}$ set equal to zero.

To obtain the homogeneous solution, $\mu_{h}$, we follow the steps that lead to (18). This gives

$$
\begin{aligned}
\mu_{h}(\boldsymbol{x}) & =\mu\left(\boldsymbol{x}_{o}\right) \exp \left\{-\int_{\boldsymbol{x}_{o}}^{\boldsymbol{x}} \boldsymbol{A}^{-1} \nabla \boldsymbol{A} \cdot d \boldsymbol{x}^{\prime}\right\} \\
& \left.=\mu\left(\boldsymbol{x}_{o}\right) \exp \left\{-\int_{\boldsymbol{x}_{o}}^{\boldsymbol{x}}[B(\nabla \cdot \boldsymbol{u}) \mathbf{1}+2 \boldsymbol{\epsilon}]^{-1}[B \nabla(\nabla \cdot \boldsymbol{u})+2 \nabla \cdot \boldsymbol{\epsilon}] \cdot d \boldsymbol{x}(2)\right\}\right)
\end{aligned}
$$

The total solution is again given by

$$
\mu=\mu_{h}+\mu_{p},
$$

with $\mu_{h}$ and $\mu_{p}$ given in (27) and (25), respectively. Again, the full solution requires $\mu\left(\boldsymbol{x}_{o}\right)$ to be known.

5.2.1. Solvability Conditions Carrying out the operations above again requires two important assumptions that may not be satisfied in practice. These are:

$$
\begin{array}{ll}
\operatorname{det} \boldsymbol{A} & \neq 0, \\
\nabla \times\left[\boldsymbol{A}^{-1} \nabla \boldsymbol{A}\right]=0 .
\end{array}
$$




\section{Plane Stress Deformations of an Incompressible Material}

In this section we show that plane stress deformations of an incompressible material may be treated as a special case of (c), above.

\subsection{Plane stress assumptions}

Plane stress is used to treat bodies that are relatively thin in one direction, say the $z$ direction. Traction free boundary conditions on the top and bottom of the thin sheet motivate the assumption:

$$
\sigma_{x z}=\sigma_{y z}=\sigma_{z z}=0 .
$$

Symmetry of the stress tensor also implies $\sigma_{z x}=\sigma_{z y}=0$. Assuming that (31) holds throughout the material tells us that $\partial_{z} u_{x}=\partial_{z} u_{y}=0$ througout the material. Thus knowing the in-plane displacement components along any plane of the sheet (say $z=0$ ) gives us the displacements through the thickness of the sheet. The momentum equations in the $x$ and $y$ directions thus reduce to:

$$
\begin{aligned}
& -\partial_{x} p+2 \partial_{x}\left(\mu \epsilon_{x x}\right)+2 \partial_{y}\left(\mu \epsilon_{x y}\right)=\rho \partial_{t t} u_{x} \\
& -\partial_{y} p+2 \partial_{x}\left(\mu \epsilon_{y x}\right)+2 \partial_{y}\left(\mu \epsilon_{y y}\right)=\rho \partial_{t t} u_{y} .
\end{aligned}
$$

The momentum equation in the $z$ direction is satisfied identically provided $\partial_{z} p=0$.

In plane stress, however, $p$ can be determined in terms of the in plane strains as follows. Using $\sigma_{z z}=0$ in the constitutive equation (3) gives:

$$
p=2 \mu \epsilon_{z z} .
$$

The incompressibility condition (5), however, tells us

$$
\epsilon_{z z}=-\left(\epsilon_{x x}+\epsilon_{y y}\right) .
$$

Using (35) and (34) in (32) and (33) gives us:

$$
\begin{aligned}
& \partial_{x}\left(2 \mu\left(\epsilon_{x x}+\epsilon_{y y}\right)\right)+2 \partial_{x}\left(\mu \epsilon_{x x}\right)+2 \partial_{y}\left(\mu \epsilon_{x y}\right)=\rho \partial_{t t} u_{x} \\
& \partial_{u}\left(2 \mu\left(\epsilon_{x x}+\epsilon_{y y}\right)\right)+2 \partial_{x}\left(\mu \epsilon_{y x}\right)+2 \partial_{y}\left(\mu \epsilon_{y y}\right)=\rho \partial_{t t} u_{y} .
\end{aligned}
$$

Equations (36) and (37) are identical to the $2 D$ restriction of equation (7), provided we take

$$
\lambda=2 \mu .
$$

Thus, case (c) above applies to plane stress deformations of an incompressible material, with $B=2$, and all deformations assumed to be two-dimensional. In particular, the matrix $\boldsymbol{A}$ becomes:

$$
\boldsymbol{A}=2\left(\epsilon_{x x}+\epsilon_{y y}\right)\left[\begin{array}{ll}
1 & 0 \\
0 & 1
\end{array}\right]+2\left[\begin{array}{ll}
\epsilon_{x x} & \epsilon_{x y} \\
\epsilon_{y x} & \epsilon_{y y}
\end{array}\right] .
$$




\section{Analytical Examples}

In this section we consider the examples of determining the shear modulus distribution given a measured strain field in a compressible body. We consider both quasistatic strain fields and a time dependent strain field. The strain fields considered are similar to the examples given in [13], where they are shown to lead to non-unique modulus distributions under the assumption of incompressible material behavior.

\subsection{Three dimensional quasi-static homogeneous deformation}

Here we consider:

$$
\begin{aligned}
& u_{x}=\epsilon x \\
& u_{y}=-\epsilon y / 2, \\
& u_{z}=-\epsilon z / 2,
\end{aligned}
$$

where $\epsilon$ is constant. Since $\nabla \cdot \boldsymbol{u} \equiv \operatorname{tr} \boldsymbol{\epsilon}=0$, we can use the results from case $(b)$ above, with $\lambda=0$. Equation (11) shows that $F_{b}=0$, since $\lambda \nabla \cdot \boldsymbol{u}=0$ and $\partial_{t t} \boldsymbol{u}=0$. Thus, $\mu_{p}=0$. Further, $\boldsymbol{\epsilon}=$ constant implies $\nabla \cdot \boldsymbol{\epsilon}=0$, and so (18) gives us

$$
\mu(\boldsymbol{x})=\mu_{h}(\boldsymbol{x})=\mu\left(\boldsymbol{x}_{o}\right) .
$$

That is, a constant strain field implies a constant modulus distribution, under the assumption that the material behaves as a compressible elastic solid.

It is interesting to compare the conclusion above with the corresponding result for an incompressible material model. By contrast to above, under the assumption that the material is incompressible the same strain field leads to the conclusion (c.f. [13] for the derivation in $2 D)$ :

$$
\mu_{\text {incompressible }}(\boldsymbol{x})=f(x)+g(y, z) .
$$

Here $f(x)$ and $g(y, z)$ are arbitrary undetermined functions. Thus, we can conclude much more about the shear modulus in a compressible material than we can in an incompressible material.

\subsection{Time dependent deformation}

We now consider the displacement field:

$$
\begin{aligned}
& u_{x}=0 \\
& u_{y}=A \sin (k x) \cos (\omega t) \\
& u_{z}=0
\end{aligned}
$$

Again, the results of case (b) apply with $\lambda=0$. Equations (15), (18) and (19) lead to (see appendix, eqns (A.7) and (A.9) for details):

$$
\mu=\frac{\rho \omega^{2}}{k^{2}}+\left[\mu\left(\boldsymbol{x}_{o}\right)-\frac{\rho \omega^{2}}{k^{2}}\right] \frac{\cos \left(k x_{o}\right)}{\cos (k x)} .
$$


If it is known that $\mu\left(\boldsymbol{x}_{o}\right)=\frac{\rho \omega^{2}}{k^{2}}$, then we obtain

$$
\mu=\frac{\rho \omega^{2}}{k^{2}} \text {. }
$$

Alternatively, we note that (48) implies that the modulus becomes infinite on lines where $\cos (k x)=0$. If we were to require that $\mu(\boldsymbol{x})$ be everywhere nonsingular, then we are forced to take $\mu\left(\boldsymbol{x}_{o}\right)=\frac{\rho \omega^{2}}{k^{2}}$ and so again arrive at (49).

\subsection{Two dimensional quasi-static homogeneous deformation}

$$
\begin{aligned}
& u_{x}=\epsilon x \\
& u_{y}=-\epsilon y, \\
& u_{z}=0,
\end{aligned}
$$

where $\epsilon$ is constant. Calculations identical to those above again lead to the conclusion

$$
\mu(\boldsymbol{x})=\mu\left(\boldsymbol{x}_{o}\right) \text {. }
$$

Again by way of contrast, the same strain field in the incompressible model leads to the conclusion [13]:

$$
\mu_{\text {incompressible }}(\boldsymbol{x})=f(x)+g(y) .
$$

Here, again, $f(x)$ and $g(y)$ are arbitrary undetermined functions.

This example leads to an interesting conclusion. Suppose we choose a modulus distribution $\mu(x, y) \neq$ constant from the set defined by (54). Now we compute a displacement field in this material using boundary conditions consistent with (50-52). If the forward computation is performed with an incompressible material model, we get exactly the displacement field (50-52). If, however, the forward computation is performed with an incompressible material model, we get a displacement field distinct from (50-52). That distinction must vanish, however, as $\lambda \rightarrow \infty$, in as much as the compressible model tends to the incompressible model in that limit. The practical effect of this recognition is made clear in the next section.

\section{Instability of reconstructions when $\lambda \rightarrow \infty$}

In this section we show by example that small changes in the given (i.e. measured) displacement field can lead to large changes in the reconstructed shear modulus distributions. To that end, we consider a slight modification of the example considered in the previous subsection.

$$
\begin{aligned}
& \epsilon_{x x}=\epsilon \\
& \epsilon_{y y}=-\epsilon(1+f(y))^{-1} \\
& \epsilon_{x y}=\epsilon_{x z}=\epsilon_{y z}=\epsilon_{z z}=0 .
\end{aligned}
$$

Here $\epsilon$ is again constant, but $f(y)>0$ is an arbitrary continuous function of the $y$-coordinate only. We note that $\epsilon_{k k}=\epsilon_{x x}+\epsilon_{y y}+\epsilon_{z z} \neq 0$, as the material is not 
incompressible. We wish to find the shear modulus distribution $\mu(\boldsymbol{x})$ in terms of $f(y)$. In this case, we shall consider $\lambda=$ known $=$ const., and so the results from case $(b)$ apply.

Equation (18) and the strain field (55-57) gives

$$
\mu_{h}(\boldsymbol{x})=\mu\left(\boldsymbol{x}_{o}\right) \frac{1+f(y)}{1+f\left(y_{o}\right)} .
$$

The particular solution follows from (15):

$$
\mu_{p}=\lambda f(y) / 2-\frac{\lambda f\left(y_{o}\right)(1+f(y))}{2\left(1+f\left(y_{o}\right)\right)} .
$$

The general solution is thus:

$$
\mu(\boldsymbol{x})=\left[\mu\left(\boldsymbol{x}_{o}\right)-\frac{\lambda f\left(y_{o}\right)}{2}\right] \frac{1+f(y)}{1+f\left(y_{o}\right)}+\frac{\lambda f(y)}{2} .
$$

In the special case that $f(y) \equiv 0$, this example reduces to the first example with a homogeneous strain field. Accordingly, equation (60) simplifies in the case $f \equiv 0$ to $\mu(\boldsymbol{x})=\mu\left(\boldsymbol{x}_{o}\right)$, as found earlier. If, however, $f(y)$ is nonzero, but merely very small, (60) simplifies to:

$$
\mu(\boldsymbol{x}) \approx \mu\left(\boldsymbol{x}_{o}\right)+\frac{\lambda}{2}\left(f(y)-f\left(y_{o}\right)\right) ; \quad f(y) \ll 1 .
$$

In the same limit, $f(y) \ll 1$, the yy component of strain in (56) becomes simply:

$$
\epsilon_{y y} \approx-\epsilon(1-f(y)) \text {. }
$$

Equation (62) shows that $f(y)$ represents the relative (or normalized) deviation of $\epsilon_{y y}$ from a constant value. Equation (61) shows that even very small fluctuations in strain can lead to very large deviations in shear modulus, $\mu$. In particular, a relative change in $\epsilon_{y y}$ of magnitude $f$, leads to a relative change in the shear modulus of magnitude $\lambda f / \mu\left(\boldsymbol{x}_{o}\right)$. When $\lambda \gg \mu$, this can represent an enormous amplification factor.

To estimate the magnitude of this amplification factor, we may consider the sound speed ratio $c_{L} / c_{s} \approx 10^{3}$. Then we are lead to the conclusion that $\lambda / \mu \approx 10^{6}$. This means

that fluctuations in strain measurements $\left(f(y)\right.$ in the above example) of magnitude $10^{-3}$, (i.e. strain measurements accurate to one part in a thousand) can give rise to fluctuations in the reconstructed modulus of relative magnitude $10^{6} \times 10^{-3}=10^{3}$, or a factor of one thousand. This represents, indeed, an enormous amplification of measurement error based on an exact solution of this inverse elasticity problem.

\section{Computational Example with Non-trivial Strain Distributions}

Here we use finite element computational solutions of the forward elasticity problem to produce our "measured" data. Additional noise is added as indicated below. 


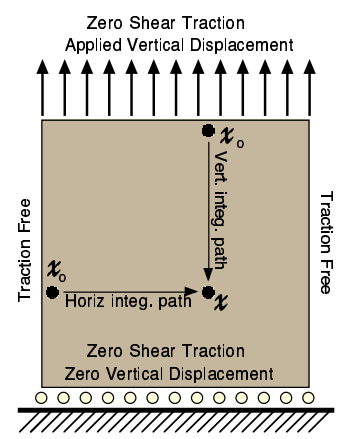

Figure 1. Boundary conditions used in the forward simulation, and two possible integration paths. For the vertical integration path, we assume we know $\mu\left(x_{o}\right)$ at each $\boldsymbol{x}_{o}$ along the top edge. For the horizontal integration path, we assume we know $\mu\left(x_{o}\right)$ at each $\boldsymbol{x}_{o}$ along the left edge.

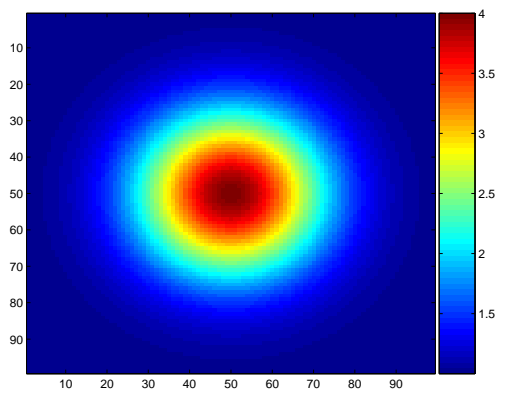

Figure 2. The reference shear modulus distribution.

\subsection{Forward Solution}

The problem modeled is tension of a thin, inhomogeneous square sheet, occupying the region $(0,1) \times(0,1)$. The boundary conditions selected are indicated in Figure 1. Figure 2 shows the "input" shear modulus distribution. It is a discretization of a Gaussian inclusion on a uniform background. In the forward computation, both the shear modulus and the displacement components are represented by bilinear interpolations on a uniform finite element mesh consisting of $101 \times 101$ nodes, or, $100 \times 100$ elements.

\subsection{Reconstruction}

We consider plane stress deformations of an incompressible material, which is a special case of case (c), and is treated in Section 6. The deformation we consider here is quasistatic. Thus the particular solution $\mu_{p}=0$, and $\mu(x, y)=\mu_{h}(x, y)$. We evaluate $\mu_{h}$ using equation (27), with $\boldsymbol{A}$ given by (32).

To evaluate strains and their derivatives, we use second order central differences to differentiate the given displacement fields $u_{x}$ and $u_{y}$ on a regular grid. The integral indicated in equation (27) is evaluated by the rectangle rule. In principle, with perfect data, the integration path will make no difference in the evaluation of the integral. For 

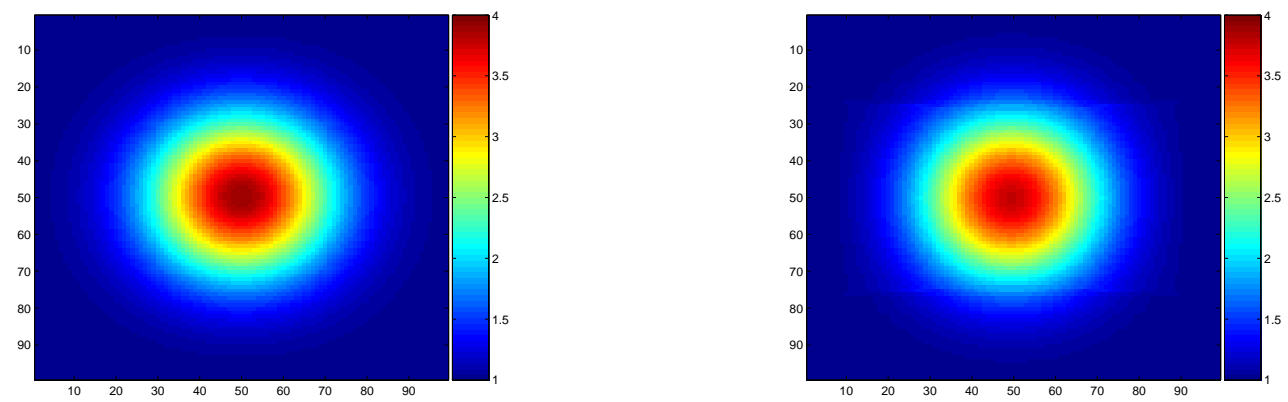

Figure 3. The reconstructed shear modulus distribution (zero noise). Left: Vertical integration paths. Right: Horizontal integration paths.

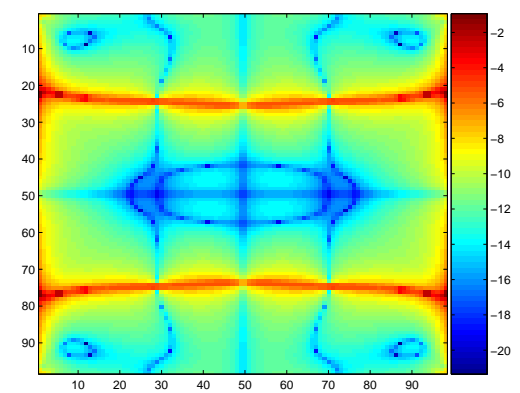

Figure 4. $\ln |\mathcal{I}|$ : The integrability condition. With perfect data and zero discretization error, $\mathcal{I}=0$. Here we see the effects of discretization error on $\mathcal{I}$. Note in particular the horizontal lines across the middle, and the relatively high values of $\mathcal{I}$ on the left and right edges.

simplicity here, we choose either a horizontal integration path or a vertical integration path, as indicated in Figure 1. In each case we assume that the modulus is constant and unity along the starting integration edge, which is approximately true in this example. Any combination of vertical and horizontal paths, or even curved paths, may be taken in principle, and in the presense of noise, some degree of smoothing might result by averging the results of many such paths.

Figures 3 show the shear modulus reconstructed by this procedure, using vertical (left) and horizontal (right) integration paths. The reconstructions are within $2-3 \%$ of the exact solution, the precise value depending on the norm used to measure the error. This is approximately the maximum level of accuracy that may be expected given the discretization error inherent in both the forward and inverse calculations. We note a slight ridge or jump in the distribution in Figure 3 (right). This may be understood by examining the solvability condition.

The solvability condition (30) may be evaluated pointwise. In principle, with exact strain fields, the left hand side of (30) should be identically zero. With discretely computed fields, and fields with noise, (30) is no longer satisfied. We define the 
integrability, $\mathcal{I}$ as:

$$
\mathcal{I}=\nabla \times\left[\boldsymbol{A}^{-1} \nabla \boldsymbol{A}\right] .
$$

When $\mathcal{I} \neq 0$, the reconstruction will depend upon the choice of the integration path from $\boldsymbol{x}_{o}$ to $\boldsymbol{x}$. Figure 4 shows $\ln |\mathcal{I}|$, pointwise within the domain. We note the greatest "sensitivity" to error along the left and right edges, and through two thin horizontal bands, about $1 / 3$ and $2 / 3$ of the way down the domain.

\subsection{Effects of Random Noise}

To investigate the effects of noise on the reconstruction, we added delta correlated, uniformly distributed, zero mean random numbers to the computed strain fields. The magnitude of the noise field is measured in terms of the signal-to-noise ratio, defined as:

$$
\begin{aligned}
\text { snr } & =\frac{\|\boldsymbol{\epsilon}\|}{\| \text { noise } \|} \\
\|\boldsymbol{\epsilon}\|^{2} & =\frac{1}{m(\Omega)} \int_{\Omega} \epsilon_{i j} \epsilon_{i j} d \Omega \\
\| \text { noise } \|^{2} & =\frac{1}{m(\Omega)} \int_{\Omega} \text { noise }^{2} d \Omega .
\end{aligned}
$$
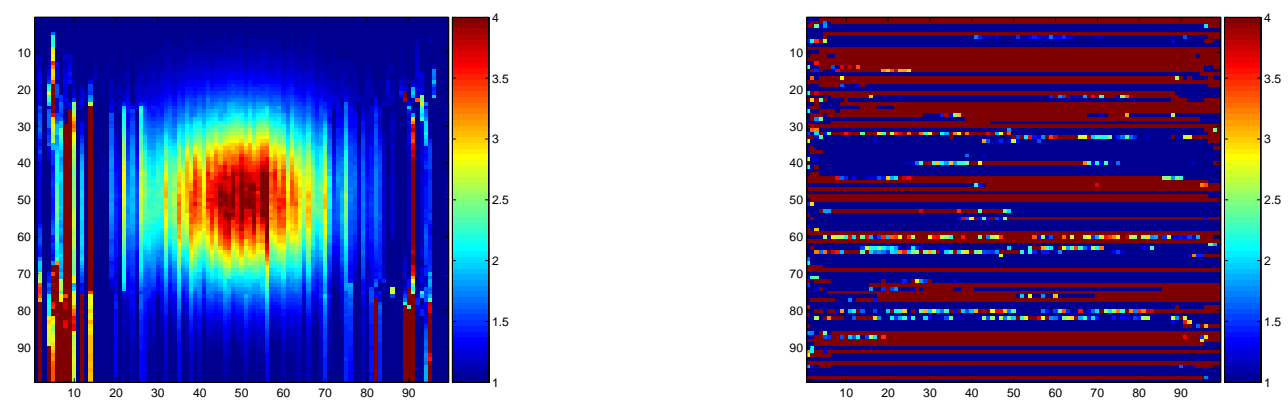

Figure 5. The reconstructed shear modulus distribution from noisy strains. Left: Vertical integration paths. Right: Horizontal integration paths. Error introduced at one point in the reconstruction tends to be propagated all along the integration path thereafter.

The reconstructions obtained for an $s n r=100$ are shown in Figure 5, left and right, for horizontal and vertical integration paths, respectively. Clearly the vertical path gives the more stable result. The integrability condition is shown in Figure 6 for this case. Comparing Figure 5 (b) to Figure 6 shows that the effect of the noise is to introduce errors at locations where the integrability $\mathcal{I}$ is high. This error tends to be propagated along the integration path. Figure 6 shows that $\mathcal{I}$ is high all along the left edge, and so error introduced there at the start of the horizontal integration is propagated throughout the entire image.

The quantitative impact of random noise on the quality of reconstructions can be mitigated by a number of techniques. For example, using a $9 \times 9$ least-squares smoothing 


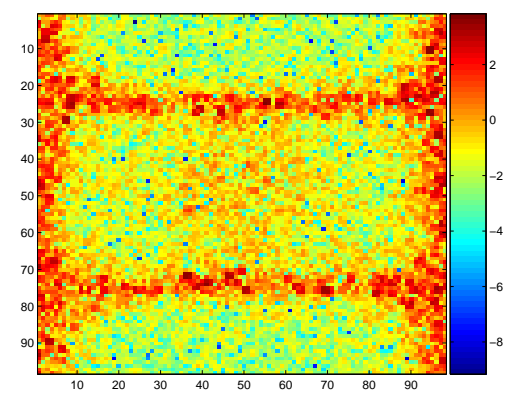

Figure 6. $\ln |\mathcal{I}|$ for noisy data. Note the ring of high $\mathcal{I}$ which corresponds to the introduction of error in the reconstructions. $\mathcal{I}$ is quite high all along the left edge, which implies that the reconstruction based on horizontal integration is doomed from the start.

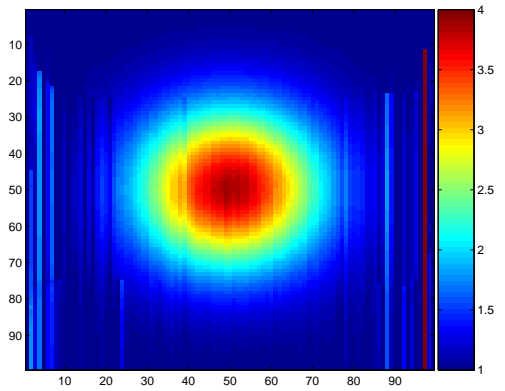

Figure 7. Reconstructed modulus from noisy strains. Noisy strains were first filtered with a $9 \times 9$ smoothing filter before modulus reconstruction with vertical integration.

filter on the strain fields yields the reconstruction shown in Figure 7. Before smoothing, this strain field was precisely the same strain field used in the previous example.

\section{Discussion and Conclusions}

We presented some exact solutions of the elastic inverse problem given measured displacement (or strain) fields. The assumptions used were:

(i) The displacement field is given. If time dependent, $\boldsymbol{u}$ and $\partial_{t t} \boldsymbol{u}$ are given at one instant in time.

(ii) linear, isotropic, compressible, elastic solid.

(iii) Either the Lame' constant $\mu$ is known, or $\lambda$ is known, or the Poisson's ratio is known.

(iv) The density (if the displacements are time dependent) is known.

We applied the exact solution derived here to some simple examples in both $2 D$ and $3 D$, in which all computations could be carried out completely analytically. The exact solution contains only one arbitrary multiplicative constant, in stark contrast to the 
gross non-uniqueness apparent in the case the material is modeled as an incompressible solid [13].

One example in particular shows a large mismatch in the Lame' parameters where $\lambda \gg \mu$ leads to a high sensitivity of the reconstructed shear modulus to the measured strains. This example modulus distribution illustrates how the compressible model can distinguish modulus distributions that the incompressible model cannot. It also demonstrates that while the compressible model can distinguish those distributions in principle, it cannot do so in practice because of its sensitivity to fluctuations in the measured strains.

Finally, we applied the reconstruction formula to a strain field computed by the finite element method. This example shows the applicability our exact solution to nontrivial strain fields. It was also used to highlight the role of noise and the impact of the choice of integration path in the presence of noise. We demonstrated how the integrability condition corresponds to which integration paths are stable or unstable.

We conclude that the reconstruction formulas derived above would be useful in practice for two classes of applications: (i) $2 D$ or $3 D$ reconstructions for compressible materials in which $\lambda / \mu=O(1)$; (ii) $2 D$ plane stress reconstructions for incompressible materials. In both cases, the full vector displacement field needs to be available to apply the formulas derived above.

\section{Acknowledgments}

This work was supported in part by NSF, BSF, DOD-CDMRP-BCRP.

\section{Appendix A. Evaluation of $\mu_{h}$ and $\mu_{p}$ in example}

Using displacement field (45-46) in the strain displacement relations (1) gives us the (plane) strain tensor:

$$
\boldsymbol{\epsilon}=\frac{1}{2} A k \cos (k x) \cos (\omega t)\left[\begin{array}{ll}
0 & 1 \\
1 & 0
\end{array}\right] .
$$

To evaluate $\mu_{h}$ by eqn (18), we need to evaluate the vector $\nabla \cdot \boldsymbol{\epsilon}$ and the tensor $\boldsymbol{\epsilon}^{-1}$. These are:

$$
\begin{aligned}
\nabla \cdot \boldsymbol{\epsilon} & =-\frac{1}{2} A k^{2} \sin (k x) \cos (\omega t)\left\{\begin{array}{l}
0 \\
1
\end{array}\right\}, \\
\boldsymbol{\epsilon}^{-1} & =\frac{2}{A k \cos (k x) \cos (\omega t)}\left[\begin{array}{ll}
0 & 1 \\
1 & 0
\end{array}\right] .
\end{aligned}
$$

Substituting (A.2) and (A.3) into (18) and simplifying gives us:

$$
\begin{aligned}
\mu_{h}(\boldsymbol{x}) & =\mu\left(\boldsymbol{x}_{o}\right) \exp \left[\int_{\boldsymbol{x}_{o}}^{\boldsymbol{x}} k \tan \left(k x^{\prime}\right)\left\{\begin{array}{l}
1 \\
0
\end{array}\right\} \cdot d \boldsymbol{x}^{\prime \prime}\right] \\
& =\mu\left(\boldsymbol{x}_{o}\right) \exp \left\{\int_{\boldsymbol{x}_{o}}^{\boldsymbol{x}} k \tan \left(k x^{\prime}\right) d x^{\prime}\right\}
\end{aligned}
$$


Some exact solutions for inverse elasticity

$$
\begin{aligned}
& =\mu\left(\boldsymbol{x}_{o}\right) \exp \left\{\log \left(\cos \left(k x_{o}\right)\right)-\log (\cos (k x))\right\} \\
& =\mu\left(\boldsymbol{x}_{o}\right) \frac{\cos \left(k x_{o}\right)}{\cos (k x)} .
\end{aligned}
$$

The particular solution $\mu_{p}$ given in equation (15) requires $\partial_{t t} \boldsymbol{u}$. Evaluating this vector from (45-46) gives

$$
\partial_{t t} \boldsymbol{u}=-A \omega^{2} \sin (k x) \cos (\omega t)\left\{\begin{array}{l}
0 \\
1
\end{array}\right\} .
$$

We then use (A.4), (A.7) and (A.8) in (15) and simplify to find:

$$
\mu_{p}=\frac{\rho \omega^{2}}{k^{2}}\left[1-\frac{\cos \left(k x_{o}\right)}{\cos (k x)}\right] .
$$




\section{References}

[1] Sarvazyan A, Rudenko O, Swanson S, Fowlkes J and Emelianov S 1998 Shear Wave Elasticity Imaging: A New Ultrasonic Technology of Medical Diagnostics Ultrasound in Medicine and Biology 24(9) 1419-35

[2] Goss S A, Johnston R L and Dunn F 1978 Comprehensive compilation of empirical ultrasonic properties of mammalian tissues Journal of the Acoustical Society of America 64(2) 423-457

[3] Van Houten E, Paulsen K, Miga M, Kennedy F and Weaver J 1999 An Overlapping Subzone Technique for MR-Based Elastic Property Reconstruction Magnetic Resonance in Medicine 99 $779-786$

[4] Weaver J, Van Houten E, Miga M, Kennedy F and Paulsen K 2001 Magnetic Resonance Elastography Using 3D Gradient Echo Measurements of Steady-state Motion Medical Physics 28(8) $1620-1628$

[5] Doyley M M, Meaney P M and C B J 2000 Evaluation of an iterative reconstruction method for quantitative elasticity Physics in Medicine and Biology 45 1521-1540

[6] Oberai A, Gokhale N, Doyley M and Bamber J 2004 Evaluation of the Adjoint Equation Based Algorithm for Elasticity Imaging Physics in Medicine and Biology 49(13) 2955-2974

[7] Oberai A, Gokhale N and Feijoo G 2003 Solution of Inverse Problems in Elasticity Imaging Using the Adjoint Method Inverse Problems 19 297-313

[8] Fung Y C 1977 A first course in continuum mechanics 2nd ed (Englewood Cliffs, NJ: Prentice Hall)

[9] Manduca A, Oliphant T, Dresner M, Mahowald J, Kruse S, Amromin E, Felmlee J, Greenleaf J and Ehman R 2001 Magnetic resonance elastography: Non-invasive mapping of tissue elasticity Med. Image Anal. 5(4) 237-254

[10] Oliphant T, Manduca A, Ehman R and Greenleaf J 2001 Complex-valued stiffness reconstruction for magnetic resonance elastography by algebraic inversion of the differential equation Magn. Reson. Med 45(2) 299-310

[11] Oliphant T, Ehman R and Greenleaf J 2002 in Imaging of complex media with acoustic and seismic waves vol 84 of Top Appl Phys pp 277-294

[12] McLaughlin J R and Yoon J R 2004 Unique identifiability of elastic parameters from time dependent interior displacement measurement Inverse Problems 20(1) 25-45

[13] Barbone P E and Gokhale N 2004 Elastic modulus imaging: On the uniqueness and nonuniqueness of the elastography inverse problem in 2d Inverse Problems 20(1) 283-296 